\title{
Influence of the Datum Definition on the Accuracy of Horizontal Geodetic Control Networks for Engineering Objects
}

\author{
Nedim Tuno, Admir Mulahusić, Jusuf Topoljak \\ Faculty of Civil Engineering, University of Sarajevo, Sarajevo, Bosnia and Herzegovina \\ E-mail:nedim_tuno@gf.unsa.ba
}

Received: 24 November 2018; Accepted: 16 December 2018; Available online: 15 March 2019

\begin{abstract}
For the construction of various civil engineering structures, particular care should be taken in the perspective of geodetic control. Therefore, the design and implementation of the geodetic network deserves special attention. This paper is focused on various aspects of datum definitions for tested micro-triangulation network. It was shown that the geometrical distribution of datum points in a minimally constrained solution has a great impact on the accuracy of the geodetic network. Estimates of the accuracies of individual station $y$ - $x$ coordinates, error circles and error ellipses, obtained by free adjustment, where the datum selection was independent of errors in fixed coordinates, revealed much better quality of control points.

Keywords: Geodetic network; Micro-triangulation; Minimally constrained adjustment; Free adjustment; Error ellipse.
\end{abstract}

\section{Introduction}

Engineering construction requires a joint participation of experts from various fields, including those from the field of geodesy [1-5]. During construction, survey activities are conducted during different phases, starting with the production of the large-scale engineering plan (map), stakeout, to the monitoring of eventual displacement and deformations of the structure during its exploitation [6].

The realization of geodetic control networks is one of the most important tasks for surveying engineers. Such networks allow all necessary works to be carried out. For example, control network represents the basis for stakeout process. Through the stake-out process, geometry of the designed structure is transferred onsite - both in horizontal and vertical sense. During such a task it is important to know the required precision of the stakeout; which is defined through the permitted deviations for the measurements and geometrical tolerances connected with the structure's location. On the basis of the coordinate errors for control geodetic network, and the errors in stakeout element measurements, it is possible to predict the accuracy that will be achieved during the stakeout of the structures' individual points [7]. Selection of the geodetic network datum, networks configuration and precision of measurements, directly affects the accuracy of its points' coordinates.

The geometry of the network plays an important role in ensuring highly reliable results [8].

Coordinates are convenient and useful when defined in a proper reference frame: a datum. The datum of a geodetic network is defined through a subset of its points, which remain congruent in time at a certain significance level. With a given datum we may compute positions and their covariance matrix. A geodetic control network consists of points and elements to be measured in three-dimensional space. It is necessary to define a coordinate reference system (datum) for the estimation procedure when using least squares adjustment with observation equations. When using condition equations, a reference coordinate system is not needed, because they express the geometrical relationships between elements of the survey without using a coordinate system [9].

Global and local position accuracy of the control points is expressed through standard deviation in the direction of the coordinate axis or total positional error. The problem with this kind of accuracy prediction is that it does not provide an insight into predicted accuracy of control points within any direction - a factor which is usually critical [6]. In this article the horizontal accuracy of the control points is analyzed through absolute error ellipses and circular error probability. In terms of a concrete example of the engineering 2D micro-triangulation network, the uncertainty of the points depending on the selection of the datum of geodetic network, was tested and analyzed.

\section{Datum problem of the geodetic network}

Four parameters define the datum for a two-dimensional coordinate system: two translation parameters, one rotation parameter, and one scale parameter. The actual geodetic measurement may include some of these 
parameters. For example, a measured distance in a network can define the scale parameter of the datum. The remaining datum parameters are determined by adding a sufficient number of constraints to the least squares problem. The datum defect of the observational system is identified with the remaining datum parameters. In case when the constraint equations supply only the necessary and sufficient number and types of datum definitions, the estimation is said to be minimally constrained. If more constraint equations are added than necessary and sufficient, the estimation is over-constrained [9].

For example, in 2D traverse survey, in which distances and angles are usually measured, if no bearing of a line is known, there will be 3 datum defects as a result of no bearing and no fixed or constrained $(y, x)$ coordinates. If the traverse is now done with a bearing of a line known, there will be 2 datum defects for lack of fixed or constrained $(y, x)$ coordinates. One of the consequences of datum defects is that they make it impossible to solve least squares adjustment of survey network where coordinates are the unknown parameters [10].

Overview of the datum defects for trigonometric network, carried out by triangulation and trilateration, is given in Table 1.

Table 1. Datum defects of trigonometric networks

\begin{tabular}{llll}
\hline $\begin{array}{l}\text { Type of the geodetic } \\
\text { network }\end{array}$ & Observations & Parameters & Defect \\
\hline Triangulation & $\begin{array}{l}\text { Horizontal directions and/or } \\
\text { horizontal angles } \\
\text { Horizontal directions and/or } \\
\text { horizontal angles and at least one } \\
\text { distance }\end{array}$ & $\begin{array}{l}\text { Two translations, one rotation, one } \\
\text { scale factor } \\
\text { Tistance }\end{array}$ & 4 \\
Trilaterationslations, one rotation & Two translations, one rotation & 3 \\
\hline
\end{tabular}

\section{Accuracy analysis of the geodetic network adjustment}

In the process of adjustment, the Gauss-Markov model is used. This model is consisted of a functional and a stochastic part. The functional part of this model is given by the expression [7]:

$$
\hat{l}=l+v=A \hat{x}
$$

where, $\hat{l}$ is vector of adjusted measurement values; $l$ is vector of measured values - observations; $v$ is vector of measurement corrections; $A$ is configuration matrix; and $\hat{x}$ is vector of unknowns.

The stochastic part of Gauss-Markov model is given by Eq. 2:

$$
K_{l l}=\sigma_{0}^{2} Q_{l l}=\sigma_{0}^{2} P^{-1}
$$

where, $K_{l l}$ is matrix of variance-covariance of measurement; $\sigma_{0}^{2}$ is a priori variance factor; $Q_{l l}$ is matrix of measurement co-factor; and $P$ is matrix of measurement weight.

The matrix of the co-factor of adjusted unknowns is there given by the following expression [7]:

$$
Q_{\hat{x} \hat{x}}=\left(A^{T} P A\right)^{-1}=N^{-1}
$$

In a free 2D geodetic network is necessary to determine the inverse of a singular matrix $N$. To find the inverse of the singular matrix $N$, the so-called matrix of datum/data conditions $B$ is formed, i.e. the pseudo-inverse matrix of $N$ is determined [11].

The basic criterion for assessing the network quality and measurement accuracy is the positional uncertainty of control points. Positional uncertainty is usually defined by standard deviations of point coordinates (Fig. 1a):

$$
s_{x}=s_{0} \sqrt{Q_{\hat{x} \hat{x}}}
$$

where $s_{0}$ is reference standard deviation a posteriori.

These values do not provide complete information regarding the accuracy of the points' location. The uncertainty of the position is likely not known in any direction other than those of the coordinate axes. It is much better if the uncertainty of the horizontal position of a point is expressed in a graphical format by the error ellipse (Fig. 1c). In practice it is common to calculate the error ellipse with a 5\% significance level $(\alpha=0.05)$.

Elements of the error ellipse — sizes of semi-major and semi-minor axes ( $a$ and $b$ ) and the orientation of the major axis (angle $\Theta$ ), are calculated according to Eq. 5: 


$$
\begin{aligned}
& \operatorname{tg} 2 \Theta=\frac{2 q_{x y}}{q_{x x}-q_{y y}} \\
& a=s_{0} \cdot \sqrt{\frac{q_{x x}+q_{y y}+\sqrt{\left(q_{x x}-q_{y y}\right)^{2}+4 q_{x y}{ }^{2}}}{2}} \\
& b=s_{0} \cdot \sqrt{\frac{q_{x x}+q_{y y}-\sqrt{\left(q_{x x}-q_{y y}\right)^{2}+4 q_{x y}{ }^{2}}}{2}}
\end{aligned}
$$

where $q_{x x}, q_{y y}$ and $q_{x y}$ are elements of the matrix $N$ [12].

The calculations according to Eq. 5 produce standard error ellipse. The chance that the point actually lays within the standard error ellipse is 39\%. The method of obtaining the 95\% error ellipse depends on whether the variancecovariance matrix of the population is unknown [13].

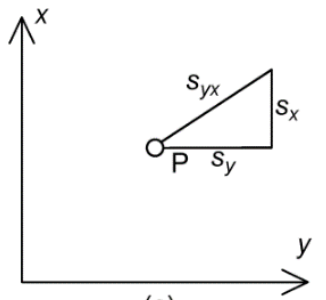

(a)

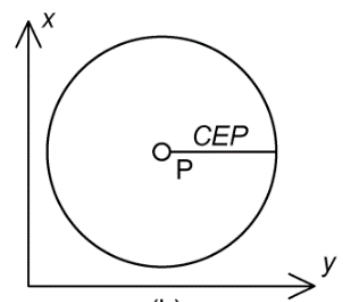

(b)

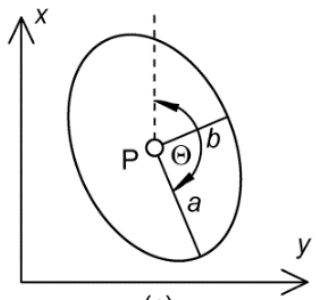

(c)

Figure 1. Accuracy estimation a posteriori: (a) standard deviations in the directions of the coordinate axis and total positional error; (b) error circle; (b) error ellipse

To express the predicted uncertainty of two-dimensional position of control points, error circles (Fig. 1b) can be used with an appropriate level of confidence; this is referred to as CEP (Circular Error Probable) [6]. The radius of the standard circle which incorporates $50 \%$ of the points is calculated by Eq. 6 :

$C E P=0.59(a+b) \pm 3 \%$, for $b / 3<a<3 b$

An error circle with 95\% confidence level is determined by Eq. 7:

$$
C E P_{95 \%}=2.08 \cdot C E P
$$

\section{Empirical case study}

Testing and analysis of the data in this study were carried out on the micro-triangulation network, located in the area of the Faculty of Civil Engineering, Sarajevo, Bosnia and Herzegovina (Fig. 2). The network is located in a densely built area, covered by trees and other high vegetation.

\subsection{Establishment of the micro-triangulation network}

With the aim of testing the influence of the datum selection, a pilot micro-triangulation network was established on the site. Such small networks are often used in various technical projects that are carried out in smaller areas. This type of network is mainly measured terrestrially, especially in urban areas and areas with dense and high vegetation. The establishment of the geodetic control network was carried out in three phases: design, execution and analysis.

Through the geodetic network project, it was determined where the control points would be placed and in which way it would be measured. It was initially anticipated that the network covered an area of 1 ha, and had to be defined by 8 points. Points are located at an average distance of $50 \mathrm{~m}$ (minimum $32 \mathrm{~m}$, maximum $66 \mathrm{~m}$ ). Due to the terrain configuration, construction and high vegetation, optimal geometric configuration of the geodetic network was achieved.

Measurements in the network began after the stabilization of points was completed. Measurements in the network were realized by electronic tachymeter Leica Geosystems TC600. The instrument precision according to DIN18723 is $\sigma_{u}=5 "$ for angle measurements [14]. Before measurements started, testing of the instrument was done according to the current ISO standard 17123-3. Test results confirmed declared precision. Measurements in 
the network involved the measurement of the horizontal directions and distances between all points (a total of 30 directions and 3 distances). Horizontal directions were observed in 3 sets.

Analysis of the geodetic network included the correction and reduction of field measurements, detection and removal of gross and systematic errors, adjustment of a geodetic network by the least squares method (parametric model), statistical testing of estimated measurement residuals, and analysis of the accuracy of obtained results.

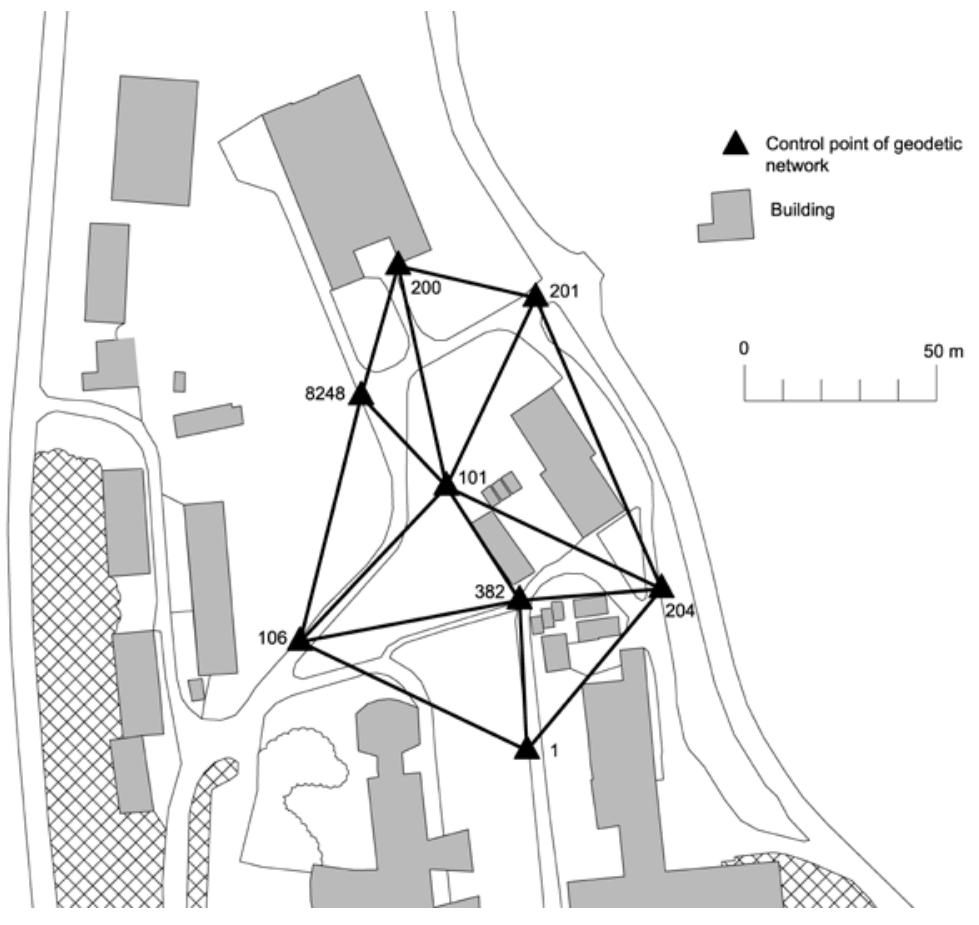

Figure 2. Tested micro-triangulation network

\subsection{Adjustment of the micro-triangulation network}

In order to determine the network in space, it is necessary to know a minimum number of parameters required for its positioning according to pre-defined coordinate systems. Since the intent of this study was to examine the accuracy of the control points — dependent on the adopted datum — four adjustments with different definitions of datum parameters of geodetic control were conducted.

The first, second and third adjustment were performed using a conventional method, i.e., definition of the network datum was done with a minimally constrained solution. In the first adjustment procedure the coordinates of the point 1 and point 106 were fixed (a measured distance between these points defined the scale parameter of the datum), giving the necessary (minimal) number of network parameters which were taken as error-free. This procedure was repeated with other two combinations of fixed points $(382,101)$ and $(200,201)$. Finally the free network adjustment was carried out, i.e., defining the optimum datum, without the introduction of points' errorfree coordinates. The algorithms of the aforementioned adjustment of the geodetic networks were provided in section 3 and various literatures $[15,16]$. The basic indicators of the accuracy analysis of the four adjustment methods are:

1) Reference standard deviation $s_{0}=2.3$

2) Standard deviation of the measured horizontal direction $s_{H}=6.6^{\prime \prime}$.

\subsection{Datum definition and its influence on the accuracy of micro-triangulation network}

In section 4.2 it was shown that the selection of the datum of the geodetic network doesn't affect the standard deviations $s_{0}$ and $s_{H}$. With the aim to investigate the impact of datum parameters on the quality of points' coordinates, the error ellipse of control points were analyzed herein for all three adopted datums, as well as error circles and standard deviations of points’ coordinates (Table 2 and Table 3).

In the case of the geodetic network with the datum definition by minimally constrained solution, correlation of obtained error ellipses and fixed points is evident. Points that are closer to the chosen origin of the coordinate system (fixed points) have smaller horizontal deviations, and the deviations of points that are further from the origin are higher. The worst results were obtained in the case when points 200 and 201 defined the datum (Fig. 3c and Fig. 4c). Ratio of minimum and maximum areas of error ellipses is approximately 1:9 in that case. Better results (approximately 30\%) were obtained when the datum was defined by the points 1 and 106 (Fig. 3a and Fig. 
4a), but the ratio of minimum and maximum areas of error ellipses is still 1:8. Slightly better results were obtained in the third definition of the datum, by fixing the coordinates of points 382 and 101 (Fig. 3b and Fig. 4b). Therefore, with this kind of datum choice, the relative relationship between the points of the network cannot be maintained. In other words, homogeneity and isotropy of their accuracy could not be achieved.

The obtained parameters of network accuracy, resulted from the coordinates of geodetic network defined by the optimal datum (free adjustment), show more balanced positional strength of all control points than in three previous cases (Fig. 3d and Fig. 4d).

In the case of a free geodetic network, a significantly better homogeneity of obtained accuracy was achieved (ratio of minimum and maximum area of error ellipse is 1:1), as well as isotropy (the ratio of average minor and major semi-axis of the ellipse is 1:1). The average positional deviation $s_{y x}$ in this case is 1.5 to 2.5 times smaller than the corresponding values obtained by minimally constrained solutions. The area of the average error ellipse is 4 to 14 times smaller. Similar results were obtained for the error circles. Therefore, a more realistic assessment of the accuracy of the control points was achieved, while satisfying all requirements.

Table 2 Indicators of the geodetic network quality (standard deviations of coordinates and positional standard deviations)

\begin{tabular}{|c|c|c|c|c|c|c|c|c|c|c|c|c|}
\hline \multirow{2}{*}{$\begin{array}{l}\text { Point } \\
\text { number }\end{array}$} & \multicolumn{3}{|c|}{$\begin{array}{l}\text { Minimally constrained } \\
\text { solution (fixed points: } \\
1 \text { and 106) } \\
\end{array}$} & \multicolumn{3}{|c|}{$\begin{array}{l}\text { Minimally constrained } \\
\text { solution (fixed points: } 382 \\
\text { and 101) }\end{array}$} & \multicolumn{3}{|c|}{$\begin{array}{l}\text { Minimally constrained } \\
\text { solution (fixed points: } \\
200 \text { and 201) } \\
\end{array}$} & \multicolumn{3}{|c|}{ Free adjustment } \\
\hline & $\begin{array}{l}S_{y} \\
{[\mathrm{~mm}]}\end{array}$ & $\begin{array}{l}S_{X} \\
{[\mathrm{~mm}]}\end{array}$ & $\begin{array}{l}S_{y x} \\
{[\mathrm{~mm}]}\end{array}$ & $\begin{array}{l}S_{y} \\
{[\mathrm{~mm}]}\end{array}$ & $\begin{array}{l}S_{X} \\
{[\mathrm{~mm}]}\end{array}$ & $\begin{array}{l}S_{y x} \\
{[\mathrm{~mm}]}\end{array}$ & $\begin{array}{l}S_{y} \\
{[\mathrm{~mm}]}\end{array}$ & $\begin{array}{l}S_{x} \\
{[\mathrm{~mm}]}\end{array}$ & $\begin{array}{l}S_{y x} \\
{[\mathrm{~mm}]}\end{array}$ & $\begin{array}{l}S_{y} \\
{[\mathrm{~mm}]}\end{array}$ & $\begin{array}{l}S_{X} \\
{[\mathrm{~mm}]}\end{array}$ & $\begin{array}{l}S_{y x} \\
{[\mathrm{~mm}]}\end{array}$ \\
\hline 1 & - & - & - & 1 & 3 & 3 & 4 & 7 & 8 & 1 & 1 & 2 \\
\hline 106 & - & - & - & 2 & 2 & 3 & 4 & 6 & 7 & 1 & 1 & 2 \\
\hline 204 & 2 & 3 & 3 & 3 & 1 & 3 & 4 & 5 & 6 & 1 & 1 & 2 \\
\hline 382 & 1 & 2 & 2 & - & - & - & 3 & 5 & 6 & 1 & 1 & 1 \\
\hline 8248 & 2 & 2 & 4 & 2 & 2 & 3 & 1 & 3 & 3 & 1 & 1 & 2 \\
\hline 101 & 2 & 2 & 3 & - & - & & 2 & 3 & 4 & 1 & 1 & 1 \\
\hline 200 & 4 & 4 & 6 & 2 & 4 & 5 & - & - & - & 1 & 1 & 2 \\
\hline 201 & 4 & 4 & 5 & 2 & 4 & 4 & - & - & - & 1 & 1 & 2 \\
\hline Average & 2.5 & 2.8 & 3.8 & 2.0 & 2.7 & 3.5 & 3.0 & 4.8 & 5.7 & 1 & 1 & 2.3 \\
\hline Max & 4 & 4 & 6 & 3 & 4 & 5 & 4 & 7 & 8 & 1 & 1 & 2 \\
\hline Min & 1 & 2 & 2 & 1 & 1 & 3 & 1 & 3 & 3 & 1 & 1 & 1 \\
\hline
\end{tabular}

Table 3. Indicators of the geodetic network quality (error circles and error ellipses)

\begin{tabular}{|c|c|c|c|c|c|c|c|c|c|c|c|c|c|c|c|c|}
\hline \multirow{3}{*}{$\begin{array}{l}\text { Point } \\
\text { number }\end{array}$} & \multicolumn{4}{|c|}{$\begin{array}{l}\text { Minimally constrained } \\
\text { solution (fixed points: } \\
1 \text { and 106) }\end{array}$} & \multicolumn{4}{|c|}{$\begin{array}{l}\text { Minimally constrained } \\
\text { solution (fixed points: } \\
382 \text { and 101) }\end{array}$} & \multicolumn{4}{|c|}{$\begin{array}{l}\text { Minimally constrained } \\
\text { solution (fixed points: } \\
200 \text { and 201) }\end{array}$} & \multicolumn{4}{|c|}{ Free adjustment } \\
\hline & \multirow{2}{*}{$\begin{array}{l}\Theta \\
{\left[{ }^{\circ}\right]}\end{array}$} & $a$ & $b$ & CEP & \multirow{2}{*}{$\begin{array}{l}\Theta \\
{\left[{ }^{\circ}\right]}\end{array}$} & $a$ & $b$ & CEP & $\Theta$ & $a$ & $b$ & CEP & \multirow{2}{*}{$\begin{array}{l}\Theta \\
{\left[^{\circ}\right]}\end{array}$} & $a$ & $b$ & CEP \\
\hline & & \multicolumn{3}{|c|}{$[\mathrm{mm}]$} & & \multicolumn{3}{|c|}{$[\mathrm{mm}]$} & {$\left[{ }^{\circ}\right]$} & \multicolumn{3}{|c|}{$[\mathrm{mm}]$} & & \multicolumn{3}{|c|}{$[\mathrm{mm}]$} \\
\hline 1 & - & - & - & - & 175 & 3 & 1 & 2.4 & 175 & 7 & 4 & 6.5 & 17 & 1 & 1 & 1.2 \\
\hline 106 & - & - & - & - & 50 & 3 & 2 & 3.0 & 29 & 6 & 4 & 5.9 & 86 & 1 & 1 & 1.2 \\
\hline 204 & 23 & 3 & 2 & 3.0 & 82 & 3 & 1 & 2.4 & 150 & 6 & 3 & 5.3 & 55 & 1 & 1 & 1.2 \\
\hline 382 & 162 & 2 & 1 & 1.8 & - & - & - & - & 171 & 5 & 3 & 4.7 & 134 & 1 & 1 & 1.2 \\
\hline 8248 & 136 & 3 & 2 & 3.0 & 138 & 3 & 1 & 2.4 & 12 & 3 & 1 & 2.4 & 1 & 1 & 1 & 1.2 \\
\hline 101 & 124 & 2 & 2 & 2.4 & - & - & - & - & 7 & 3 & 2 & 3.0 & 166 & 1 & 1 & 1.2 \\
\hline 200 & 170 & 4 & 4 & 4.7 & 169 & 4 & 2 & 3.5 & - & - & - & - & 4 & 1 & 1 & 1.2 \\
\hline 201 & 11 & 4 & 4 & 4.7 & 22 & 4 & 2 & 3.5 & - & - & - & - & 104 & 1 & 1 & 1.2 \\
\hline Average & & 3.0 & 2.5 & 3.2 & & 3.3 & 1.5 & 2.9 & & 5.0 & 2.8 & 4.6 & & 1 & 1 & 1.2 \\
\hline Max & & 4.0 & 4.0 & 4.7 & & 4.0 & 2.0 & 3.5 & & 7.0 & 4.0 & 6.5 & & 1 & 1 & 1.2 \\
\hline Min & & 2.0 & 1.0 & 1.8 & & 3.0 & 1.0 & 2.4 & & 3.0 & 1.0 & 2.4 & & 1 & 1 & 1.2 \\
\hline
\end{tabular}




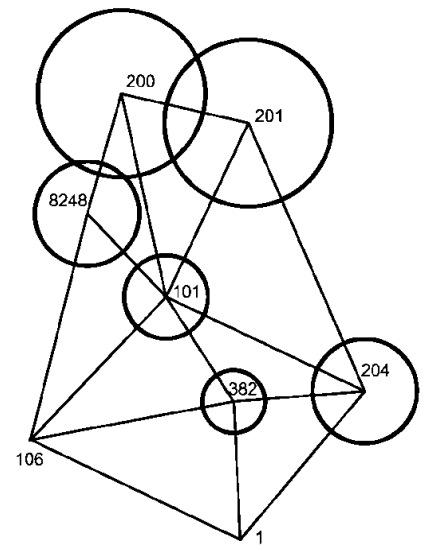

(a)

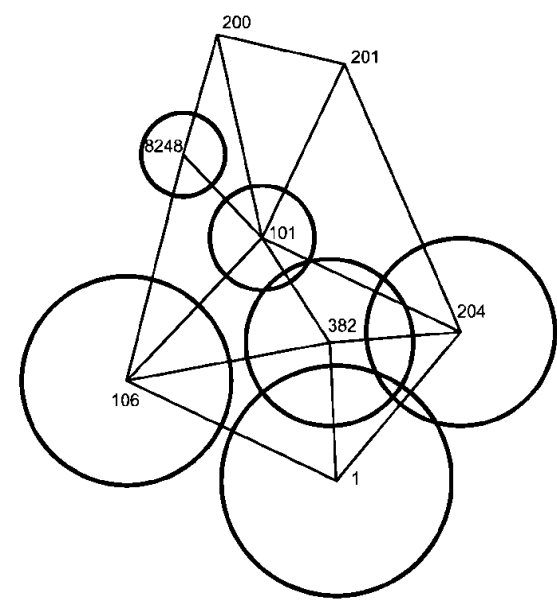

(c)

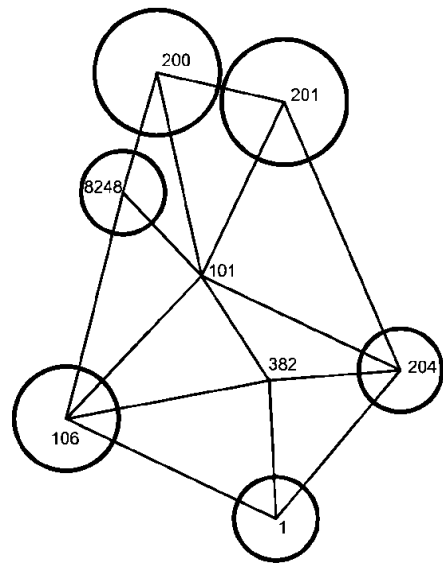

(b)

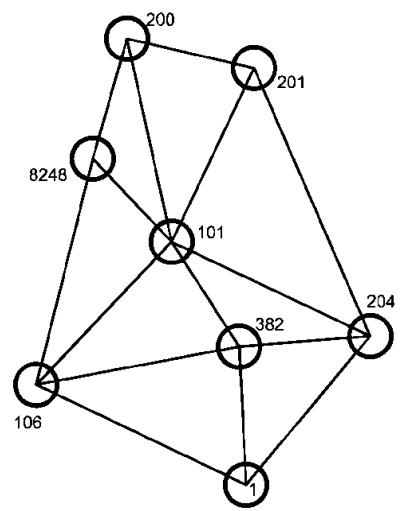

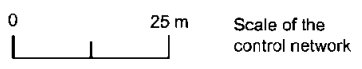

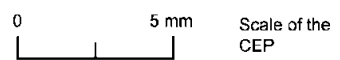

Figure 3. The influence of the selected geodetic network datum on the accuracy of control points represented by error circles: (a) minimally constrained solution (1 and 106 fixed); (b) minimally constrained solution (101 and 382 fixed); (c) minimally constrained solution (200 and 201 fixed); (d) free adjustment

\section{Conclusions}

This study clearly shows that more complete information about the uncertainty of the position of control points is obtained when using error ellipse, as opposed to the usual methods of accuracy assessment in the directions of the coordinate axes or by error circles. Other than describing the uncertainty of the position of a point, a great advantage of the error ellipses lies in the fact that a visual comparison of achieved accuracy in any two points can be done.

Qualitative examination of the influences that dictate the accuracy of the micro-triangulation network has shown that the choice of the geodetic network datum has the great influence on the uncertainty of the control points. It was noted that the network that were adjusted with minimally constrained solution, cannot ensure the stringent requirements of accuracy for control points. The worst scenario is when the datum is defined by points located at the end of the network, and the distance between them is small. More reasonable results were achieved if the fixed points are located in the central part of the network. Due to the required accuracy, the geodetic network should be adjusted by defining the optimum datum. 


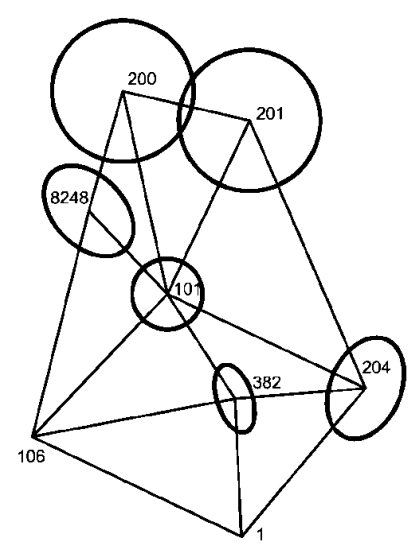

(a)

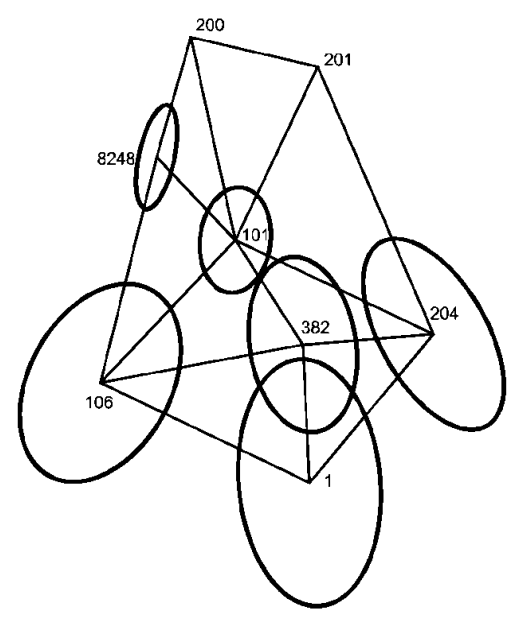

(c)

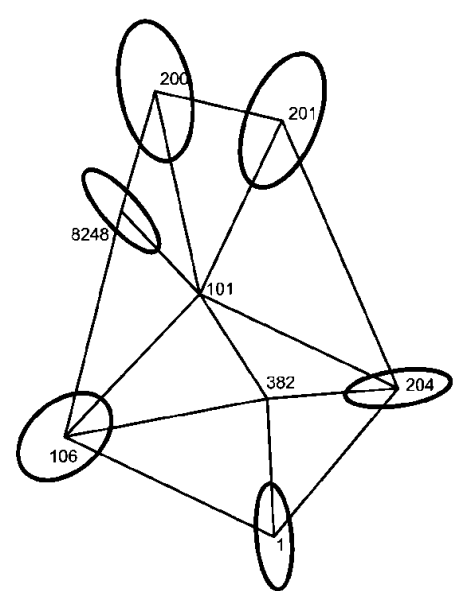

(b)

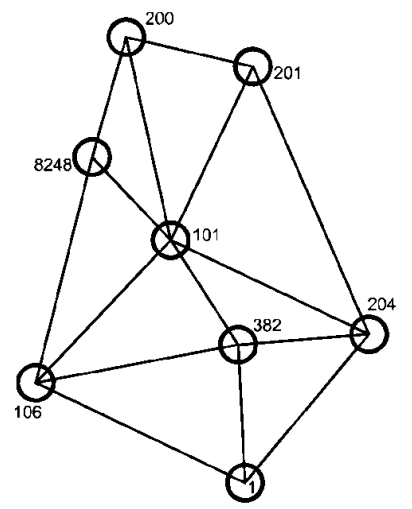

(d)

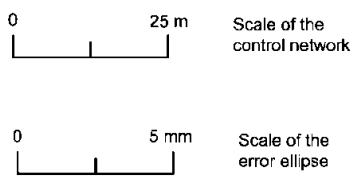

Figure 4. The influence of the selected geodetic network datum on the accuracy of control points represented by error ellipses: (a) minimally constrained solution (1 and 106 fixed); (b) minimally constrained solution (101 and 382 fixed); (c) minimally constrained solution (200 and 201 fixed); (d) free adjustment

\section{References}

[1] Tong X, Meng X, Ding K. Estimating geometric parameters of highways and railways using least-squares adjustment. Survey Review. 2010;42(318):359-374. DOI: 10.1179/003962610X12747001420582.

[2] Zrinjski M, Džapo M. Geodetic Basis of the Longest Tunnel in the Republic of Croatia. Survey Review. 2010;42(318):345-358. DOI: 10.1179/003962610X12747001420546.

[3] Lõhmus H, Ellmann A, Märdla S, Idnurm S. Terrestrial laser scanning for the monitoring of bridge load teststwo case studies. Survey Review. 2018;50(360):270-284. DOI: 10.1080/00396265.2016.1266117.

[4] Huang S, Li G, Wang X, Zhang W. Geodetic network design and data processing for Hong Kong-ZhuhaiMacau link immersed tunnel. Survey Review. 2017:1-9. DOI: 10.1080/00396265.2017.1385200.

[5] Casaca J, Braz N, Conde V. Combined adjustment of angle and distance measurements in a dam monitoring network. Survey Review. 2015;47(342):181-184. DOI: 10.1179/1752270614Y.0000000106.

[6] Tuno N, Topoljak J, Ademović N, Mulahusić A. A Simulation Analysis on the Expected Horizontal Accuracy of a Bridge Stakeout. Technical gazette. 2018;25(1):285-293. DOI: 10.17559/TV-20161115105536. 
[7] Baykal O, Tari E, Coşkun MZ, Erden T. Accuracy of point layout with polar coordinates. Journal of surveying engineering. 2005;131(3):87-93. DOI: 10.1061/(ASCE)0733-9453(2005)131:3(87).

[8] Erdogan B, Hekimoglu S. Effect of subnetwork configuration design on deformation analysis. Survey Review. 2014;46(335):142-148. DOI: 10.1179/1752270613Y.0000000066.

[9] Even-Tzur G. Datum definition for GPS networks. Survey review. 2000;35(277):475-486. DOI: 10.1179/sre.2000.35.277.475.

[10] Ogundare JO. Understanding least squares estimation and geomatics data analysis. Hoboken, New Jersey, USA: John Wiley \& Sons;2018.

[11] Božić B, Ristić K, Pejić M. Parameter estimation and accuracy analysis of the free geodetic network adjustment using singular value decomposition. Technical gazette 2014;21(2): 451-456.

[12] Mihailović K, Aleksić I. The concepts of networks in surveying. Belgrade, Serbia: Geokarata; 2008. (in Serbian)

[13] Schofield W, Breach M. Engineering surveying, 6th ed. Amsterdam, Netherlands:Elsevier Ltd.; 2007.

[14] Tuno N, Mulahusić A, Marjetič, A, Kogoj, D. Overview of development of electronic tachymeters Leica geosystems. Geodetski vestnik. 2010;54(4):643-660. (in Slovenian). DOI: 10.15292/geodetskivestnik.2010.04.643-660.

[15] Harvey BR. Practical least squares for surveyors, 3rd ed. Kesington, Australia: School of Surveying and Spatial Information Systems, University of New South Wales; 2006.

[16] Ghilani C. Adjustment Computations: Spatial Data Analysis, 5th Ed. United Kingdom: John Wiley and Sons Ltd; 2010.

(C) 2019 by the author(s). This work is licensed under a Creative Commons Attribution 4.0 International License (http://creativecommons.org/licenses/by/4.0/). Authors retain copyright of their work, with first publication rights granted to Tech Reviews Ltd. 